\title{
Hybrid
}

Revue des arts et médiations humaines

$6 \mid 2019$

L'écoute

\section{Écoute corporelle et sensibilité analogique : l'« école sonore plastique » de la Villa Arson et sa confrontation au numérique}

\section{Léa Dreyer}

\section{(2) OpenEdition}

Journals

Édition électronique

URL : https://journals.openedition.org/hybrid/548

DOI : $10.4000 /$ hybrid. 548

ISSN : 2276-3538

Traduction(s) :

Listening with the body and an analogue sensibility: the Villa Arson's "sound art" school in the digital age - URL : https://journals.openedition.org/hybrid/553 [en]

Éditeur

Presses universitaires de Vincennes

Référence électronique

Léa Dreyer, «Écoute corporelle et sensibilité analogique : l'« école sonore plastique » de la Villa Arson et sa confrontation au numérique », Hybrid [En ligne], 6 | 2019, mis en ligne le 04 novembre 2019, consulté le 13 avril 2022. URL : http://journals.openedition.org/hybrid/548 ; DOI : https://doi.org/ 10.4000/hybrid.548

Ce document a été généré automatiquement le 13 avril 2022

Revue Hybrid 


\title{
Écoute corporelle et sensibilité analogique : l'« école sonore plastique » de la Villa Arson et sa confrontation au numérique
}

\author{
Léa Dreyer
}

1 L'intérêt majeur de l'exposition Le Temps de l'Écoute ${ }^{1}$, en 2011, tient de la volonté de ses commissaires Éric Mangion et Jean-Marc Avrilla de dresser un inventaire transversal des pratiques sonores et musicales sur la Côte d'Azur depuis les années 1950, en faisant émerger des liens théoriques et esthétiques intergénérationnels comme ébauche préalable à un travail critique. L'occasion aussi de voir apparaître dans ce paysage artistique un berceau pédagogique et son héritage, prenant source dans l'enseignement sonore de Lars Fredrikson à la Villa Arson. Communauté d'écoute la plus "durable » dans une histoire sonore régionale fondée sur « des expériences non concertées ou fugaces $^{2} "$, le studio son de Lars Fredrikson a en effet fédéré un ensemble d'artistes Éléonore Bak, Isabelle Sordage, Ludovic Lignon, Pascal Broccolichi - autour d'une transmission d'un art de l'écoute qui s'inscrit dans la continuité naturelle des expériences radicalement non musicales des premiers plasticiens sonores de la seconde moitié du $\mathrm{xx}^{\mathrm{e}}$ siècle, dont Fredrikson fait partie. Historiquement, le studio fait office de premier pôle sonore dans une école d'art en France, bien avant sa généralisation dans les années 2000.

2 En 1970, Lars Fredrikson entre en tant que professeur de gravure à l'école de la Villa Arson, alors École des arts décoratifs de Nice (ENAD) ${ }^{3}$. Il y devient professeur d'audiovisuel l'année suivante, et travaille aux côtés de Bob Guiny. Il est difficile de donner une date précise à la création du studio son: on peut tout juste estimer qu'à partir de 1986, alors que Christian Bernard ${ }^{4}$ prend la direction de la Villa, il acquiert son officialité en devenant un enseignement à part entière. À sa création et sous la direction de Lars Fredrikson, le studio son de la Villa Arson est exclusivement réservé à une pratique du «son plastique». Il n'est pas une plateforme d'appui à des projets 
multimédia et encore moins un lieu de création musicale. En cela, c'est un véritable terrain de transmission d'une conception sonore alors très mal comprise par les institutions de formation artistique françaises, à une époque où la discipline plastique sonore peine à se démarquer de celle de la grammaire compositionnelle musicale.

Entre 1993 et 1998, dans les écoles d'art françaises, on ne considérait pas encore vraiment le sonore comme un territoire plastique (les Lars Fredrikson étaient rares). Mon cursus a été de fait une valse houleuse entre mes professeurs favorables à cette démarche et ceux qui considéraient que ma place était à l'IRCAM (Le dernier endroit où j'aurais voulu étudier 5 !).

3 Laissant la part belle à la rencontre entre la recherche scientifique et un «être au monde " poétique, l'écoute fredriksonienne fait appel à la notion d'audition dans une acception large : un effort perceptif vers un ensemble de flux énergétiques plus ou moins proches et leur exploration corporelle, subjective et intuitive. La pédagogie sonore de Lars Fredrikson trouve ses fondements dans une conception plastique et inclusive de la vibration: elle ne comprend pas seulement, comme le fait R. Murray Schafer, le son «sonnant ", audible. Ce dernier n'est qu'une manifestation sensible d'un large spectre vibratoire - comprenant infrasons et ultrasons, ainsi que tout le spectre électromagnétique.

4 L'étude des sons en histoire de l'art est frappée par la difficulté ontologique que représente la fragilité du médium sonore vis-à-vis des autres domaines plastiques. Cette fragilité est redoublée dans la pratique du son de Lars Fredrikson et de ses étudiants par des facteurs qui rendent son traitement complexe. Ils recoupent notamment : un refus, souvent, de la captation d'installations sonores et la nécessité d'une écoute corporelle, vécue ; l'impossibilité de générer une documentation visuelle qui pourrait nourrir les archives; la prédilection pour le médium analogique, et, conséquemment, une difficulté à la « reconstitution » de travaux sonores. C'est donc en prenant acte de l'attitude pédagogique de Lars Fredrikson dans sa transmission de l'écoute et de la pratique sonore que nous pouvons le mieux comprendre son héritage, caractérisé aujourd'hui par des profils artistiques qui, malgré leur variété, se rejoignent tous dans une écoute corporelle, « méthexique ${ }^{6}$ », spatiale et plastique.

Étudiante, je savais qu'il était un bon artiste, mais c'est effectivement sa pédagogie naturelle qui nous a tous portés. Celle de l'écoute principalement, écoute de soimême, écoute de son propre espace, de l'espace vécu, de l'espace plastique ensuite que nous devions développer, chacun à notre façon. Écoute de l'autre également. Seule l'expérience sensible peut nous faire avancer dans ce type de pratique, et c'est ce vers quoi il nous invitait?

5 C'est, par ailleurs, dans des événements fédérateurs, dans diverses postures pédagogiques et lieux de transmission que cette notion d'«école sonore plastique " fredriksonienne trouve aujourd'hui un relai dans un cadre institutionnel et géographique élargi.

\section{« Imageophobie » et esthétique du seuil : une posture pédagogique ténue}

6 La présence officielle du studio son au sein de l'école et son inclusion dans la formation des étudiants en tant qu'enseignement à part entière n'ont pas immédiatement participé d'une grande sensibilisation, à échelle de l'institution, aux questions de la pratique plastique sonore. Lorsque Sordage présente son diplôme en 1990, le jury le lui 
refuse en prétextant que son travail ne relève pas d'une discipline plastique mais d'une pratique musicale. Elle soumet les mêmes pièces l'année suivante, en soulignant leur appartenance légitime aux arts plastiques, et obtient son diplôme avec les félicitations. Ce quasi-rejet du phénomène sonore en école d'art trouve alors son origine dans la conception très ténue et radicale qu'en a Lars Fredrikson.

7 «Imageophobe ", l'écoute fredriksonienne rejette violemment les structures linguistiques et visuelles, l'artiste empêchant la production de toute forme de musique dans le studio son et se défaisant d'une approche conceptualiste alors prédominante. Son enseignement est celui d'un déconditionnement lent. Il s'agit avant tout de s'interroger sur l'activité discrète de l'écoute, d'en prendre conscience comme action subjective et partagée, non définitive. L'écoute fredriksonienne est une production continue d'attention et de doute, en convolution avec les phénomènes vibratoires. En cela, Fredrikson rejoint la notion de "caractère sonore " tel que Max Neuhaus la définit : perçu par un sens auditif inné, non culturel, le «caractère sonore » donne au son « une strate supplémentaire de signification, qui s'ajoute au langage verbal ${ }^{8}$ ». Cette approche s'explique aussi par la complicité qu'entretiennent Lars Fredrikson et certains de ses étudiants avec la pratique de la poésie sonore, auprès de laquelle ils trouvent la compréhension réciproque qu'il leur est alors difficile d'obtenir auprès des plasticiens « traditionnels».

$8 \mathrm{Si}$, pendant ses années d'enseignement au studio son, il ne réalise pas de séances d'écoute en extérieur - dans la tradition des soundwalks initiée par Max Neuhaus dans les années 1970 et généralisée dans la pédagogie de Murray Schafer ${ }^{9}-$, le studio son est essentiellement un lieu de partage philosophique et d'écoute. Ces séances d'échanges, collectives et individuelles, ont certainement permis à ses étudiants de comprendre les données constitutives d'un exercice qualitatif de l'écoute dans sa spatialité et ses nuances perceptives. Fredrikson leur fait découvrir des pièces d'Alvin Lucier, de Max Neuhaus ou de Steve Reich. On y apprend l'importance de l'événement sonore non tonitruant, dans l'efficience discrète ${ }^{10}$ des variations sonores infimes.

On me demande assez souvent pourquoi je n'augmente pas plus l'intensité sonore des installations que j'expose, comme si ce qui est visible ne garantissait pas assez l'authenticité et la source des sons qui se propagent. Je préfère toujours jouer avec les illusions des images sonores et me concentrer sur les manifestations que créent les propagations spatiales, plutôt que de hausser sommairement le volume des amplis pour essayer de mettre tout le monde d'accord sur ce qu'écouter veut $\operatorname{dire}^{11} \ldots$

\section{Un « suprématisme analogique ${ }^{12}$ »: opérations de transduction et de rémanences}

9 Dès les années 1960, Lars Fredrikson réalise pièces électromécaniques, collages, peintures, qui trouvent, au-delà de leurs médiums hétéroclites, leur fondement dans sa pensée des forces invisibles. Benhamou parle d'ailleurs de "suprématisme analogique » pour définir la pratique fredriksonienne: la déclinaison intuitive d'une manière de traduire, sous des formes variées, les tensions produites par "l'énergie du vide», les structures complexes de ce qu'on ne voit pas. Pour Fredrikson, le son n'existe pas de manière autonome, il est l'un des registres de l'invisible et est compris dans ses relations au monde des fréquences. Ce mode de pensée, écho de la philosophie pythagoricienne - qui décrit l'ubiquité de sons inaudibles de l'échelle tellurique à 
l'échelle sidérale ${ }^{13}$-, est relayé par le suprématisme et les premières phases du constructivisme russe dont Fredrikson revendique l'influence. Ils ont en commun le pressentiment d'un monde fondé sur la synchronicité des rythmes de l'univers et des rythmes intérieurs, une connexion cénesthésique, intime, avec les énergies élémentaires.

10 C'est autour de cette pensée énergétique qui prend en compte un « déjà-là » inaudible, invisible, que se réunissent aujourd'hui les artistes issus du studio son. Les premières rencontres de Pascal Broccolichi avec Lars Fredrikson ont lieu autour de l'écoute d'enregistrements de radio VHF - radio très haute fréquence équipée sur les bateaux que Broccolichi capte en mer. Cette pratique de la transduction représente aujourd'hui une part importante de la pratique de l'artiste, qui construit sur cette base des « méta paysages» complexes. Lignon traduit quant à lui des micro événements électromagnétiques générés sur la base des hasards "programmés » de ses circuits électroniques par des témoins lumineux.

11 C'est également en s'appuyant sur divers exercices de transduction, d'équivalences et de rémanences que naît le langage sonore de cette communauté de l'écoute, vocabulaire plastique qui s'établit en véritable grammaire de la construction spatiale, prenant en compte les données architecturales préexistantes que les artistes conjuguent parfois à leurs propres structures, qu'elles soient immatérielles ou «en dur».

12 Isabelle Sordage entre à la Villa Arson en 1984. Sensible aux situations d'entropie dans le règne énergétique, elle se saisit de modèles mathématiques pour aborder les phénomènes de désorganisation propres au domaine du vivant. «Les désordres et les disparitions me fascinaient à tel point que la relation que j'entretenais avec le sensible ne pouvait se satisfaire du registre figé des images et du visible imposé par l'école ces années-là̀ ${ }^{14} »$. Sordage intègre peu après son ouverture le studio son en 1987, où elle est la première étudiante. Alors que l'enseignement académique lui impose un modèle imitatif s'appuyant sur la géométrie descriptive, « suivant cette croyance qu'un travail plastique devait passer par l'apprentissage d'un espace à trois dimensions ${ }^{15}$ ", Sordage remplace les valeurs métriques qu'on lui inculque par des unités de mesure en Hertz.

D'une certaine façon, je tentais de transposer dans l'univers sonore certaines questions posées par la sculpture en termes d'espace. J'élaborais une mise en espace du son de la même manière que s'il s'agissait d'un volume avec un angle de vision particulier. Je pensais l'objet sonore par résolutions graphiques et faisais intervenir des jeux d'ombres, de surfaces, de lignes, de courbes, etc. ${ }^{16}$

13 Abstraire la ligne de sa réalité mathématique a ouvert des possibilités d'exploration de l'« espace plastique », auquel il manquait certaines références visuelles. Chez Isabelle Sordage ou Ludovic Lignon, il s'agit de considérer l'onde directionnelle comme une ligne, et de construire cette ligne sonore comme l'on construit la géométrique euclidienne, de mettre en place un jeu d'équivalences sans entrer dans le domaine de la modélisation, donner naissance à un type de projection visuelle permettant de prendre conscience de cette architecture sonore comme réalité plastique autonome.

Les plasticiens du son de la Villa Arson, comme Pascal Broccolichi ou Isabelle Sordage, mettent fréquemment en place des protocoles visant à révéler l'aspect graphique de la vibration sonore. Ces transpositions et rémanences, si elles ne relèvent pas d'une pratique de l'écoute subjective et individuelle - leur aspect final est le résultat d'une fixation graphique autonome défaite par son immobilité des phénomènes vibratoires - 
donnent lieu à un travail de décryptage visuel et haptique, et se déclinent selon des procédés "archaïques" qui se font les échos des travaux d'Ernst Chladni, dont les outils ont révélé l'« image » sonore et le caractère spectaculaire des motifs ondulatoires à la fin du XVIII siècle.

Fig. 1

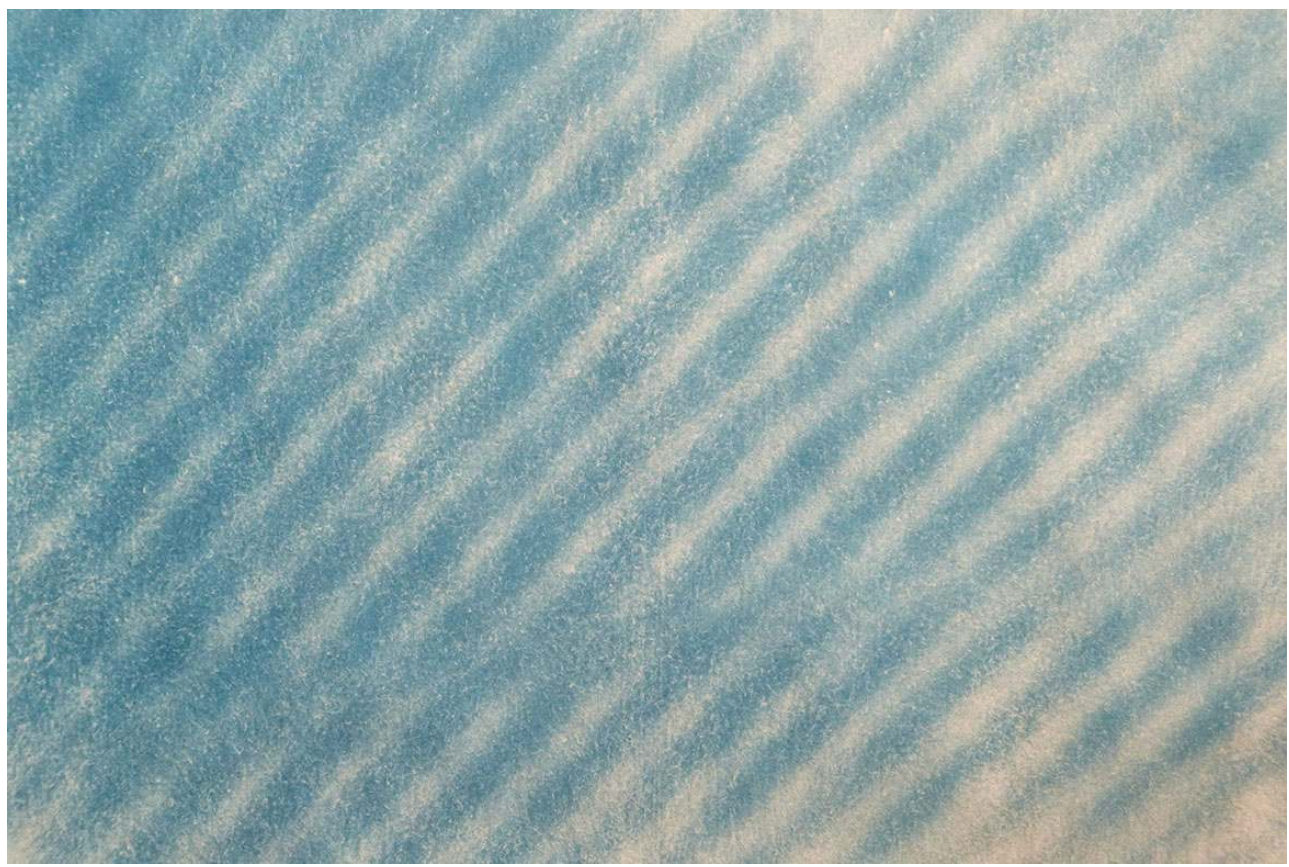

Isabelle Sordage, Sans titre (série Fibra-Frequency), 2014. Impression sur feuille photosensible des motifs générés à la surface de l'eau par des infrasons.

(c) Isabelle Sordage

\section{Le « corps antenne » : expérience corporelle de la matérialité sonore}

Comme Yann Paranthöen les sculpte et Knud Viktor les peint, Lars Fredrikson et ses étudiants envisagent les sons dans leur plasticité, et opèrent le déplacement de l'esthétique du geste créatif depuis le registre visuel vers le domaine de l'invisible vibratoire. D'un niveau perceptif à l'autre, ils trouvent leur matière première dans le médium analogique et invitent à un véritable exercice d'exploration corporelle, tout autant dans le processus créatif que dans le vécu de l'œuvre.

C'est le rapport au vivant qui est inévitable avec l'analogique; le rapport à sa plasticité, à sa mobilité, à sa physicalité, à sa malléabilité. Tout ceci reste intact avec l'analogique. Même un souffle est riche si on le laisse vivre. Chez Lars le souffle est très important par exemple. [...] L'analogique est une empreinte du vivant ${ }^{17}$.

Éléonore Bak fait ses études artistiques à Cologne. À travers une pratique poussée de la performance, elle débute en 1985 celle du son, grâce à une bourse de recherche qui l'amène à se rendre à la Villa Arson. Là, elle étudie l'interaction corporelle avec les signaux sonores continus et travaille principalement sur un générateur de signaux appareil de mesure et de synthèse électronique. Auprès de Lars Fredrikson, elle perfectionne une écoute basée sur des principes écologistes et contextuels. Sa thèse, 
Habiter l'in-vu : formes de visualisations sonores ${ }^{18}$, est un témoignage précieux d'une pensée de l'écoute héritée de sa pratique de la performance et de sa complicité avec Lars Fredrikson.

En reprenant la théorie de l'objet sonore de Pierre Schaeffer, fondée sur les trois données entrant en compte dans la grammaire de l'écoute de la musique concrète - le sonore, le musical, et le sens -, Bak choisit de substituer au caractère «musical » le caractère "plastique ${ }^{19}$ ». La donnée "plastique», pour Éléonore Bak, est interdépendante du sonore et de son sens. Elle en constitue l'aspect mouvant, non répétable, situationnel. Le "sens" qui en découle est interactionnel, aperceptif et subjectif. Son évolution ne sert pas une intention musicale, mais une écoute orientée vers le sillage du phénomène sonore, son instabilité.

Le projet de fin d'étude de Ludovic Lignon, étudiant de Fredrikson, relève de ce transfert. Dans 1990/92 (1992), l'artiste opère le déplacement de données sonores musicales à des données sonores plastiques. Il y considère des extraits musicaux préexistants et provenant de supports $\mathrm{CD}$, vinyles et bandes magnétiques, comme une matière à part entière.

Les nombreuses formes musicales passaient simultanément par une table de mixage, ainsi détournées, sans effet ajouté ni retouche numérique. [...] Le contexte était clairement musical alors que je cumulais les couches d'enregistrement avec une volonté de " peintre $»^{20}$.

19 L'enseignement et la pratique de Lars Fredrikson nous éclairent sur la compatibilité entre son technologique et expérience sensorielle augmentée. La souveraineté du sujet, encouragée par la discrétion des dispositifs et l'invisibilité du son, amène une conception de l'écoute incorporant et dépassant les notions d'" ambiance", de " paysage » sonore. L'écoute de Fredrikson implique à la fois l'ensemble des données acoustiques d'un lieu et leur interaction avec le corps, « récepteur » sensible. Ce « corps antenne » est le fruit d'une conception écologiste de l'environnement immédiat qui prend en compte de multiples facteurs, un mode de connexion corporelle à divers phénomènes interreliés de nature complexe. Partant de techniques de concentration et d'harmonisation, le corps arrive à un stade de convolution multisensorielle où il s'imprègne, absorbe un ensemble de données relevant du domaine de la microperception. Il est la résultante d'une exposition prolongée, d'une implication aperceptive, et d'un lent "déconditionnement " à l'image, entrave à l'expérience plastique par sa mise à distance réflexive des motifs environnementaux. Sur la base d'ajustements inconscients, le corps antenne se prolonge à son environnement, dans une temporalité ralentie, trouble, une spatialité élastique ${ }^{21}$. Si Éléonore Bak développe sa méthodologie en en explorant les résonances sensibles plutôt que les impacts psychologiques, le concept de « corps antenne » qu'elle développe auprès de Fredrikson opère la jonction théorique entre une écoute technique, rationnelle, et celle, plus méditative, développée tout au long de son parcours par Pauline Oliveros, qui développe sur la base du concept global de Sonosphere ${ }^{22}$, le Quantum listening («écoute quantique »).

\section{Difficile confrontation aux technologies numériques}

20 Le parcours des plasticiens sonores issus de la Villa Arson est marqué, dans leurs années de formation, par l'explosion de l'accessibilité du numérique. Leur profil est 
d'autant plus intéressant qu'ils ont unanimement débuté leur pratique avec des outils analogiques et en ont pour certains gardé un usage régulier voire exclusif.

Là où en analogique il $\mathrm{y}$ a déperdition, en numérique il $\mathrm{y}$ a transformation de cette perdition. Lorsque nous écoutons un enregistrement - le microphone ayant pris la place des oreilles, la bande s'étant substituée à la perception - nous percevons une matière sonore préfigurée. Le fait de l'assistance de l'écoute par le microphone, puis par le casque, réduit l'espace originairement courbe en des données normées [...]. Plusieurs types de distances se manifestent: la distance culturelle (on peut reconnaître un son sans lui attribuer un sens) et sociale (mémoire collective, mémoire intime), la distance entre le vécu et le représenté. La prise en charge du son par les technologies comporte une nouvelle distance, la distance entre le réel et le virtuel $[. . .]^{23}$.

La confrontation théorique et pratique entre la méthodologie de l'écoute corporelle de Bak et celle de l'ingénierie acoustique est révélatrice de cette tendance analogique. La virtualité de la gestion et la visualisation du son par interface informatique, ainsi que la spatialisation sonore fondée sur des repères géométriques orthonormés, relèvent pour elle d'une «incarcération technologique ${ }^{24} »$. Cela nous aide à saisir les difficultés de compréhension que l'artiste a rencontrées auprès des ingénieurs, notamment quant à la complexité des micro mouvements qu'elle emploie et de la lecture de ses jacquards ${ }^{25}$. Elle parvient toutefois à confirmer l'efficacité technique de l'exploration corporelle de l'espace sonore et la validité de ses restitutions lors d'expériences menées dans une smartroom holophonique ${ }^{26}$.

\section{Fig. 2}

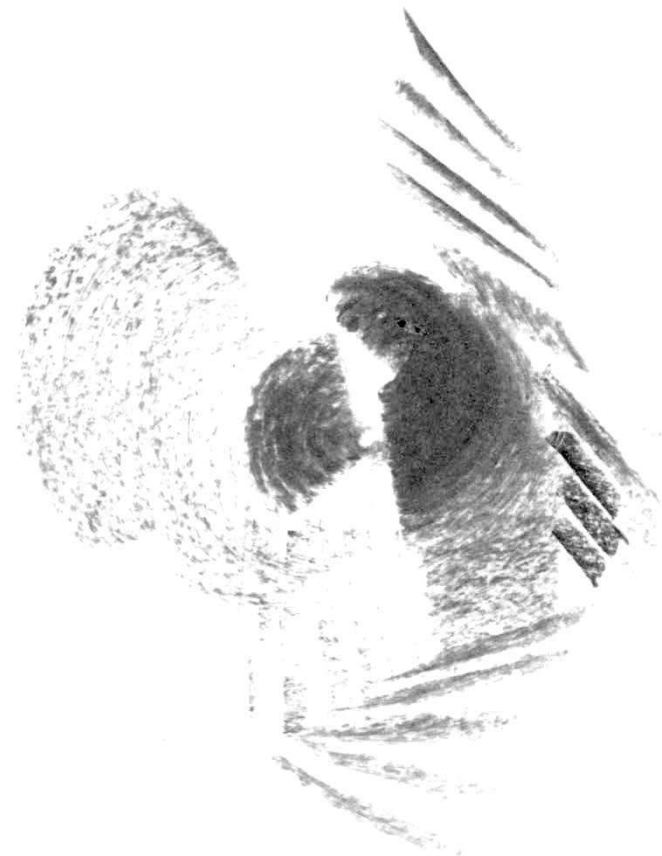

Éléonore Bak, Henri Milo (dessin d'écoute de la fontaine de Clans), 2007. Fusain sur papier, A3.

(c) Éléonore Bak 


\section{L'« école sonore » de la Villa Arson : éclatements et continuités}

Après le départ de Lars Fredrikson en 1991, Ludovic Lignon gère le studio son. Il y organise plusieurs séances d'écoute au haut-parleur et tâche d'y poursuivre l'enseignement de Fredrikson. À l'arrivée de Jérôme Joy en 1992, il devient son assistant pendant trois ans. Les changements radicaux que Joy opère dans le studio expliquent peut-être le départ de Lignon $^{27}$. Le studio, auparavant dans une salle d'envergure, déménage dans un espace insonorisé, confiné. Il est entièrement équipé numériquement, bouleversant l'ergonomie, la matérialité et la temporalité de la pratique sonore des artistes, qui travaillent désormais principalement devant l'écran. Ce changement de cadre bouleverse irréversiblement la nature de l'enseignement qui y est prodigué. Du fait de sa radicalité, la pratique sonore qui s'y est développée auprès de Fredrikson a laissé peu de traces.

Nous sommes d'une génération d'artistes, qui, animée par des préoccupations sociétales, s'est lancée dans l'expérience de courants alternatifs, notamment écologiques. [...] En réponse au marché de l'art des années 1980 en plein essor spéculatif et qui privilégiait l'objet et la peinture, nous dédaignions l'art matériel et l'empire de l'image, et nous refusions l'archivage. [...] L'art sonore, dont la valeur marchande ne représentait pas grande chose à l'époque, se dessinait comme une ouverture puissante ${ }^{28}$.

En 1995, Jean-Philippe Vienne organise l'exposition Murs du Son ; Murmures ${ }^{29}$ à la Villa Arson. Il choisit de ne pas établir la définition d'un genre artistique, mais de proposer un ensemble non exhaustif d'occurrences sonores dans le domaine de l'art. Tandis que Lars Fredrikson y présente Espace intérieur (1975-1995), ses anciens étudiants - Isabelle Sordage, Ludovic Lignon, Pascal Broccolichi - sont invités à intervenir dans le volet «Murmures». Cette exposition est la première à confirmer l'influence de la création du studio son et de l'enseignement de Lars Fredrikson auprès d'une génération de jeunes artistes. Mais un des aspects caractéristiques des héritiers de «l'école sonore " de la Villa Arson est leur propension à adopter une posture pédagogique. Plusieurs structures ont vu le jour, qui nous permettent d'envisager la transmission de l'écoute fredriksonienne dans ses réémergences les plus variées.

Parce que l'art des sons est souvent réduit aux dispositifs technologiques, parce qu'on n'a toujours pas écrit l'histoire de l'art sonore et de ses familles de pensée avec le même souci que l'art visuel, parce que les musées semblent ne pas s'en inquiéter, la pédagogie est pour moi une bonne manière de pointer des choses que l'auditeur lambda ignore, voire ne peut percevoir en peu de temps ${ }^{30}$...

En 1996, Isabelle Sordage fonde l'Atelier expérimental, installé depuis 2002 à Clans dans l'arrière-pays niçois. Il privilégie les processus de recherche et la cohabitation avec les œuvres, et développe depuis sa fondation de nombreuses interventions auprès du public. Il a par ailleurs accueilli plus d'une cinquantaine de résidences d'artistes plasticiens et musiciens, commissaires d'exposition, scientifiques ou historiens de l'art, parmi lesquels Éléonore Bak, Ludovic Lignon, Jérôme Joy, Luc Kerléo, mais aussi Pierre Laurent Cassière, Jean-Marc Lévy Leblond, Éliane Radigue ou Emmanuel Holterbach. Les résidents s'engagent à intervenir auprès de la communauté clansoise et déposer une production pour enrichir le fonds de la Villa les Vallières.

Par ailleurs, les travaux de recherche d'Éléonore Bak et son enseignement sont aujourd'hui un exemple abouti de relai de l'écoute fredriksonienne, une vision 
pédagogique plurielle et enrichie se présentant comme une véritable extension universitaire et artistique de l'«école sonore" de la Villa Arson. Aujourd'hui professeure à l'ESAL (école supérieure d'art de Lorraine), Éléonore Bak y initie en 2009 L'ARS (l'atelier de recherche sonore) ${ }^{31}$. Cadre aux projets artistiques des étudiants de l'école qui impliquent le son, L'ARS sensibilise ses participants aussi bien aux domaines techniques et scientifiques qu'à une méthodologie de l'écoute. Gravitant autour de Bak, l'atelier possède des partenariats avec le CRESSON et l'École nationale supérieure d'architecture de Grenoble, ainsi que Centrale Supélec et des institutions outre-Rhin.

Inclus dans le " pôle numérique » depuis que Jérôme Joy en a pris la direction à la suite de Lars Fredrikson en 1992, le studio son de la Villa Arson est quant à lui aujourd'hui supervisé par Pascal Broccolichi, ancien étudiant de Fredrikson ayant pleinement embrassé les possibilités offertes par les outils digitaux dans sa pratique. Si la présence de Broccolichi permet de faire le pont avec la tradition du son dans sa conception plastique, le studio est aujourd'hui majoritairement numérique, multimédia, les étudiants y trouvant souvent un appui à des projets plus globaux. Depuis sa direction par Jérôme Joy, qui l'a en partie axé vers le partage en réseau et l'écoute dans l'ère internet, le studio son s'est engagé dans une vision plus large de la pratique sonore, s'ouvrant aux questions politiques et écologiques, ainsi qu'à celles soulevées par l'économie de production. On note par ailleurs, depuis les années 2000, une réelle ouverture aux sound studies dans leur aspect transdisciplinaire, avec la multiplication de workshops auprès d'artistes invités et de nombreuses conférences.

Le rejet du tout numérique chez certains anciens étudiants de Lars Fredrikson n'est pas le fruit d'une radicalité « coquette » ou rétrograde. Il n'y a donc pas lieu d'antagoniser l'approche d'artistes qui, comme Pascal Broccolichi, ont choisi les technologies digitales. Cette pratique trouve ses fondements dans une conception de l'expérience des flux qui tâche d'explorer un "être au monde» corporel quasi-primitif et ses territoires sensibles les plus ténus. Elle est aussi manifeste de la persistance des tendances antidiscursives et anticonceptuelles de la seconde moitié $d u x^{e}$ siècle et en constitue un exemple dans le domaine du son en art, offrant un cadre méthodologique à cette exploration des énergies « du vide ».

\section{BIBLIOGRAPHIE}

Avrilla, Jean-Marc et Mangion Éric (dir.), Le Temps de l'Écoute, catalogue d'exposition, Nice, Villa Arson, 24 juin-30 octobre 2011, Dijon, Les presses du réel, 2014.

Bak, Éléonore, Habiter l'in-vu : formes de visualisations sonores, thèse dirigée par Grégoire Chelkoff, architecture, aménagement de l'espace, Université Grenoble Alpes, 2016.

Benhamou, Maurice, Lars Fredrikson, Rustrel, L'Ollave éditeur, 2019.

Davila, Thierry, De l'inframince. Brève histoire de l'imperceptible, de Marcel Duchamp à nos jours, Paris, Du Regard, 2010.

Davila, Thierry (dir.), Pascal Broccolichi. Cartographie de l'inouï, Dijon, Les presses du réel, 2012. 
Fredrikson, Lars, [cat. exp.], Lars Fredrikson, Nice, MAMAC, 16 novembre 2019-22 mars 2020], Monaco, NMNM/Mousse Publishing, 2019.

Gillon, Gaël, L'Atelier de la création, « Le corps antenne », une émission d'Isabelle Sordage, diffusée le 5 Mars 2013 sur France Culture.

Holterbach, Emmanuel, « Microphonie, paysages et trompe-l'oreille », TACET, n 3, 2014.

Hunt Vinton, Frederick, Origins in Acoustics, New Haven, Yale University Press, 1978.

Kahn, Douglas, Earth Sound Earth Signal - Energies and Earth Magnitude in the Arts, Berkeley, University of California Press, 2013.

Lignon, Ludovic. [En ligne] http://llignon.net/ [consulté le 12 avril 2018].

Nancy, Jean-Luc, À l'écoute, Paris, Galilée, 2002.

Oliveros, Pauline, « Quantum Listening : From Practice to Theory (to Practice Practice) », 1999.

[En ligne] https://soundartarchive.net/articles/Oliveros-1999-Quantum_listening.pdf, [consulté le 20 avril 2018].

Schafer, Raymond Murray (dir.), Le Paysage sonore : le monde comme musique, Marseille, Wildproject Éditions, 2010.

Sordage, Isabelle, catalogue non-publié.

\section{NOTES}

1. Nice, Villa Arson, 24 juin-30 octobre 2011, commissariat de Jean-Marc Avrilla et Éric Mangion. Avec les travaux d'Éliane Radigue, Lars Fredrikson, Isabelle Sordage, Ludovic Lignon, Pascal Broccolichi, Christian Vialard, Vincent Epplay, Jérôme Joy, Arnaud Maguet, Robert Malaval, Jean Dupuy, Thomas Köner... (Jean-Marc Avrilla, Éric Mangion (dir.), Le Temps de l'Écoute, catalogue d'exposition, Nice, Villa Arson, 24 juin-30 octobre 2011, Dijon, Les presses du réel, 2014).

2. Éric Mangion, in Jean-Marc Avrilla, Éric Mangion (dir.), Le Temps de l'Écoute, catalogue d'exposition, Nice, Villa Arson, 24 juin-30 octobre 2011, Dijon, Les presses du réel, 2014, non paginé.

3. L'École nationale des arts décoratifs de Nice prend place en 1972 sur le site de la Villa Arson. La même année, y est inauguré le CARI (le centre artistique de rencontres internationales).

4. En 1986, l'ENAD devient l'EPIAR (école pilote internationale d'art et de recherche). Christian Bernard prend alors la direction de l'ensemble des institutions de la Villa. Il oriente résolument l'activité de l'école vers l'art contemporain, le département Art remplaçant les précédents départements Communication, et Environnement. Par ailleurs, il resserre les liens entre le centre d'exposition et la pédagogie: l'enseignement à la Villa bénéficie de la présence continue d'artistes en résidence qui interviennent auprès des étudiants, ces derniers étant également confrontés à des expositions d'envergure internationale qui prennent place sur l'ensemble du site.

5. Emmanuel Holterbach, « Microphonie, paysages et trompe-l'oreille », TACET, n 3, 2014, p. 354. 6. «Selon le regard, le sujet se renvoie à lui-même comme objet. Selon l'écoute, c'est en quelque sorte en lui-même que le sujet se renvoie ou s'envoie. [...] Ou encore, en termes quasi lacaniens, le visuel serait du côté d'une capture imaginaire [...] tandis que le sonore serait du côté d'un renvoi symbolique. [...] En d'autres termes encore, le visuel serait tendanciellement mimétique, et le sonore tendanciellement méthexique (c'est-à-dire dans l'ordre de la participation, du partage ou de la contagion) »; Jean-Luc Nancy, À l'écoute, Paris, Galilée, 2002, p. 26-27.

7. Isabelle Sordage, mail du 11 juin 2018. 
8. Yehuda Safran et Zsuzsanna Gahse, Three to One. Max Neuhaus, Bruxelles, La Lettre Volée, 1996, cité dans Maria Andueza Olmedo, «Quelques réflexions sur le son et l'espace urbain », TACET, n³, 2014, p. 198.

9. «De manière plus générale, il est intéressant de noter que ces éléments de sensibilisation au sonore qui, selon Schafer font partie d'une démarche pédagogique cohérente ("l'éducation de l'oreille"), se retrouvent toujours comme fondement de la plupart des programmes actuels de formation aux pratiques du son en France, qu'elles soient techniques (par exemple, l'école Louis Lumière) ou artistiques (par exemple, les Ateliers Saint-Sabin ENSCI ou certaines écoles des beaux-arts) ». Postface de Nicolas Misdariis et Patrick Susini, in Raymond Murray Schafer (dir.), Le Paysage sonore : le monde comme musique, Marseille, Wildproject Éditions, 2010, p. 406.

10. «L'efficience sera cette façon discrète (indirecte) d'opérer en prenant appui sur les transformations silencieuses, sans faire saillir d'événement, de façon à faire croître progressivement l'effet au travers d'un déroulement. Il s'agira moins de conduire pompeusement, héroïquement - que d'induire l'effet. Efficacité me paraît désormais trop spectaculaire, "efficience" n'est attaché qu'au rendement ", François Jullien, Traité de l'efficacité, Paris, Grasset, 1996, p. 78-79. Repris dans Thierry Davila, De l'inframince. Brève histoire de l'imperceptible, de Marcel Duchamp à nos jours, Paris, Du Regard, p. 18.

11. Pascal Broccolichi, in Thierry Davila (dir.), Pascal Broccolichi. Cartographie de l'inouï, Dijon, Les presses du réel, 2012, p. 39.

12. Maurice Benhamou, «Suprématisme analogique », in Lars Fredrikson, Rustrel, L'Ollave éditeur, 2019, p. 22-26.

13. Frederick Vinton Hunt, Origins in Acoustics, New Haven, Yale University Press, 1978, p. 12.

14. Isabelle Sordage, catalogue non publié, p. 5. Certains éléments sont disponibles sur le site personnel de l'artiste. En ligne : [http://www.isabelle-sordage.fr/].

15. Isabelle Sordage, catalogue non publié, p. 5.

16. Isabelle Sordage, catalogue non publié, p. 5.

17. Isabelle Sordage, mail du 12 avril 2018.

18. Éléonore Bak, Habiter l'in-vu: formes de visualisations sonores, thèse dirigée par Grégoire Chelkoff, architecture, aménagement de l'espace, Université Grenoble Alpes, 2016.

19. Éléonore Bak, Habiter l'in-vu: formes de visualisations sonores, thèse dirigée par Grégoire Chelkoff, architecture, aménagement de l'espace, Université Grenoble Alpes, 2016, p. 225.

20. Ludovic Lignon. [En ligne] http://llignon.net/ [consulté le 12 avril 2018].

21. À ce sujet: Gaël Gillon, L'Atelier de la création, "Le corps antenne », une émission d'Isabelle Sordage, diffusée le 5 Mars 2013 sur France Culture.

22. Douglas Kahn, «Pauline Oliveros : Sonosphere », in Douglas Kahn, Earth Sound Earth Signal Energies and Earth Magnitude in the Arts, Berkeley, University of California Press, 2013, p. 174-186. Voir également Pauline Oliveros, "Quantum Listening: From Practice to Theory (to Practice Practice) ", 1999. [En ligne] https://soundartarchive.net/articles/Oliveros-1999Quantum_listening.pdf, [consulté le 20 avril 2018]..

23. Éléonore Bak, Habiter l'in-vu: formes de visualisations sonores, thèse dirigée par Grégoire Chelkoff, architecture, aménagement de l'espace, Université Grenoble Alpes, 2016, p. 261.

24. Éléonore Bak, Habiter l'in-vu: formes de visualisations sonores, thèse dirigée par Grégoire Chelkoff, architecture, aménagement de l'espace, Université Grenoble Alpes, 2016, p. 49. Expression empruntée à Tim Ingold, Une brève histoire des lignes (2007), traduction Sophie Renaut, Bruxelles, Zones Sensibles, 2011.

25. «Pendant que nous lisions notre carte selon un ordre immersif, les ingénieurs la lisaient comme un système oui/non, point par point, ligne par ligne, de gauche à droite, du haut vers le bas. Pendant que nous cherchions à reproduire la somme ressentie de ce qui faisait scène (soit : l'anachronisme des phénomènes, leur géométrie variable, leur décentrement - et le dispositif holophonique nous laissait imaginer un « jouer » simultané, continu de sons de même nature -), 
afin de dévoiler la physionomie extrêmement fine de l'ensemble (teneur plastique, intensités des émergences et disparitions, dissimulation des vides et de pleins dans le tout), les ingénieurs, eux, se consacraient essentiellement à la reproduction des mouvements, dont l'apparition/disparition franche des phénomènes sous forme de séquences ponctuellement rehaussées d'évènements instantanées. " Éléonore Bak, Habiter l'in-vu: formes de visualisations sonores, thèse dirigée par Grégoire Chelkoff, architecture, aménagement de l'espace, Université Grenoble Alpes, 2016, p. 141.

26. Salle d'étude acoustique équipée d'un ensemble de haut-parleurs permettant de recréer des environnements comprenant des sources sonores virtuelles se déplaçant dans l'espace. La smartroom de Centrale Supélec de Metz comprend un holoplayer, qui peut jouer 16 sources en simultané sur 76 haut-parleurs.

27. Jérôme Joy a une formation musicale. Il est orienté vers le partage en réseau, les possibilités offertes par le web naissant permettant alors une toute nouvelle conception de l'écoute. À son arrivée, le studio se numérise entièrement, changeant à la fois d'orientation esthétique et d'« ergonomie » de pratique sonore.

28. Éléonore Bak, Habiter l'in-vu: formes de visualisations sonores, thèse dirigée par Grégoire Chelkoff, architecture, aménagement de l'espace, Université Grenoble Alpes, 2016, p. 41

29. Nice, Villa Arson, 8 juillet- $1^{\text {er }}$ octobre 1995, commissariat de Jean-Philippe Vienne. Avec José Antonio Orts, Pascal Broccolichi, Robert Barry, Lars Fredrikson, Jérôme Joy, Ludovic Lignon, Éric Maillet, Kristin Oppenheim, etc. (Michel Bourel (dir.), Mur du son; Murmures [exp. Nice, Villa Arson, 8 juillet- $1^{\text {er }}$ octobre 1995], Nice, Villa Arson, 1996 : support CD).

30. Éléonore Bak, mail du 11 juin 2018.

31. Il s'agit évidemment d'un hommage d'Éléonore Bak à Lars Fredrikson.

\section{RÉSUMÉS}

Depuis quelques années, la réémergence de l'intérêt porté à Lars Fredrikson, pionnier des pratiques sonores expérimentales, soulève un certain nombre de problématiques liées à la question du son en art et de la pédagogie de l'écoute dans les écoles. Porteur d'une conception plastique et corporelle de l'écoute, l'enseignement de Lars Fredrikson à la Villa Arson entre 1970 et 1991 - où il crée le premier studio son dans une école d'art en France - permet d'observer la transmission auprès d'une génération d'artiste, de pratiques sonores durablement marquées par un rejet de l'approche musicale et une compréhension du son comme "matière vivante ». Ce déplacement des caractéristiques de la sculpture, et plus généralement des arts visuels, vers le domaine vibratoire, s'accompagne de la persistance d'un goût pour une " esthétique du signal », propre aux outils de l'esthétique analogique, qui apparait à plusieurs échelles dans la création chez ces artistes.

\section{INDEX}

Mots-clés : son, expériences sonores, son plastique, Villa Arson 


\section{AUTEUR}

\section{LEA DREYER}

Léa Dreyer est doctorante en histoire de l'art contemporain à l'université Paris I-Panthéon Sorbonne, où elle travaille sous la direction de Pascal Rousseau sur l'œuvre de Lars Fredrikson. Elle a travaillé sur la notion de plasticité sonore dans la pratique pédagogique et la transmission de l'écoute de Fredrikson à l'école de la Villa Arson (mémoire de master 2). Elle a été chargée de recherches dans la préparation de la première grande rétrospective de l'artiste, au MAMAC de Nice (bourse de recherche du NMNM de Monaco). En 2020, elle bénéficie de la bourse des Amis du Centre Pompidou pour réaliser la mission de recherche « Les sons plastiques de Lars Fredrikson : étude d'un corpus d'œuvres sonores et de ses modes de diffusion » sous la direction de Marcella Lista. 